\title{
The influence of concurrent training intensity on serum irisin and abdominal fat in postmenopausal women
}

\author{
Bita Azimi Rashti ${ }^{1}$, Javad Mehrabani ${ }^{2}$, Arsalan Damirchi², Parvin Babaei ${ }^{3}$ \\ ${ }^{1}$ Department of Exercise Physiology, University Campus 2, University of Guilan, Guilan, Rasht, Iran \\ 2Department of Exercise Physiology, Faculty of Physical Education and Sport Sciences, University of Guilan, Guilan, Rasht, Iran \\ ${ }^{3}$ Cellular and Molecular Research Center, Guilan University of Medical Sciences, Guilan, Rasht, Iran
}

\begin{abstract}
Introduction: Menopause seems to be among the factors related to the development of prediabetes and central obesity. Conversely, physical activity may have a major role in reducing metabolic syndrome in women of postmenopausal age.

Material and methods: Forty-eight postmenopausal active and sedentary women within the ages of 45-65 years were randomized to four groups: 1$)$ high-intensity concurrent interval exercise $(\mathrm{HCl} ; n=15)$, 2) moderate-intensity continuous concurrent exercise (MCC; $n=14)$, 3) control daily active (CDA; $n=10)$, and 4) control sedentary (COS; $n=9$ ). The subjects in the preceding training groups exercised three times per week for 50-65 min/session with high/moderate concurrent exercise for 10 weeks. The levels of serum irisin, insulin sensitivity, abdominal fat distribution (visceral and subcutaneous), and total abdominal fat were measured at baseline and post-test.

Results: This study showed that the $\mathrm{HCl}$ group had a significantly increased serum irisin $(p<0.001)$, stimulated favorable alterations in insulin sensitivity status $(p=0.003)$, and significantly decreased subcutaneous, visceral and, total abdominal fat $(p<0.001)$. Also, the insulin sensitivity status was significantly increased $(p=0.01)$ and subcutaneous abdominal fat significantly decreased in the MCC group $(p=0.018)$. However, this study's results did not find a considerable correlation between insulin sensitivity, irisin level, weight loss and abdominal fat distribution.

Conclusions: High-intensity concurrent exercise has a greater influence on improving most metabolic-related parameters than moderate-intensity concurrent exercise.
\end{abstract}

Key words: concurrent exercise, irisin, insulin sensitivity, abdominal fat, postmenopausal women.

\section{Introduction}

Increases in fat mass and weight are specifically linked to the menopausal phase [1]. Conditions such as obesity, especially the amount of abdominal fat, are often associated with cardiovascular diseases and risk factors such as plasma lipids, hyper-insulinemia, and insulin resistance [2]. Deterioration of physiological activities, associated with aging and inactivity, may also intensify during menopause [3]. Exercise, which is believed to be associated with improvements in insulin sensitivity, can aid in visceral fat reduction, especially in the central regions of the body [4]. The underlying molecular and cellular mechanism is not clearly understood, but research tells us that the muscle contractions lead to high secretions of myokines, which are assumed to mediate the positive effects of exercise [5].
As such, physical exercise stimulates the expression of the fibronectin type III domain containing 5 (FNDC5) gene, which leads to high levels of eponymous protein successors. FNDC5 is the pro-protein of irisin, a type of myokine, which engenders the transformation of white to beige adipocytes via uncoupling protein 1 [6]. Subsequently, the body's energy expenditure and thermogenesis also rise in response to the secretion of irisin. This event has an enriching effect on metabolic traits (such as decreased insulin sensitivity, high fasting blood sugar, hyperlipidemia, reduced HDL, impaired adipose tissue function) linked to overweight and obesity [6, 7].

Based on existing studies, exercise type (endurance/ resistance), exercise time (acute/chronic), exercise intensity, and personal training prior to participation in studies count as factors affecting the circulating irisin 
level. Indeed, different studies have reported physical exercise influences are significant in irisin circulation levels [8-10], while other studies show that exercise, acute and/or chronic, has no substantial effect on blood irisin level [11-13]. Therefore, the role of exercise on irisin activity has been a controversial subject [14-17]. Furthermore, inconsistent results have come into view regarding irisin concentrations caused by exercise. In another study, a significant increase in irisin levels after a session of high-intensity interval training (HIIT) was observed, while Pilates seems to have no influence on overweight women [18]. Likewise, there is very little knowledge about how high-intensity concurrent training might affect metabolic functions in the middle-aged woman's well-being.

Despite conflicting data, the effect of irisin on metabolic traits linked to obesity appears favorable. However, it must be mentioned that post-training irisin measurements in postmenopausal women were not performed. Therefore, the concrete effect of exercise in promoting expression and secretion of irisin in the human body remains uncertain. On the other hand, as hypothesized by the authors, circulating levels of myokines may fluctuate differently under the influence of different types of exercises. The selected protein was chosen because it was newly discovered as a plasma myokine. Irisin and its metabolic functions have not been previously studied in postmenopausal women.

Based on the favorable effects of aerobic and resistance training on health, the current research in- tends to examine the effects of two concurrent exercise training programs on insulin sensitivity status, serum levels of irisin and abdominal fat distribution (visceral vs. subcutaneous) and total abdominal fat in postmenopausal women.

To achieve more accurate results, two control groups were selected: one for the potential effects of daily exercises and the second to account for any additional factors. The training program lasted for ten weeks. Our hypothesis herein is that high-intensity concurrent interval exercise will have a greater influence on improving most metabolic-related parameters than moderate-intensity concurrent interval exercise.

\section{Material and methods}

\section{Participants}

One hundred and seventeen postmenopausal participants within the ages of 45-65 years were recruited to the study via notices at Rasht area City Park and clubs. Being at least one year in menopausal stage and no alcohol or drug addiction were the criteria for women to be a part of the study. All participants were matched for age and body mass index (BMI; calculated as weight in kilograms divided by the height in meters squared). Subjects of the study signed written consent, filled out a medical questionnaire, and received a thorough description of the research methods before participation in the study.

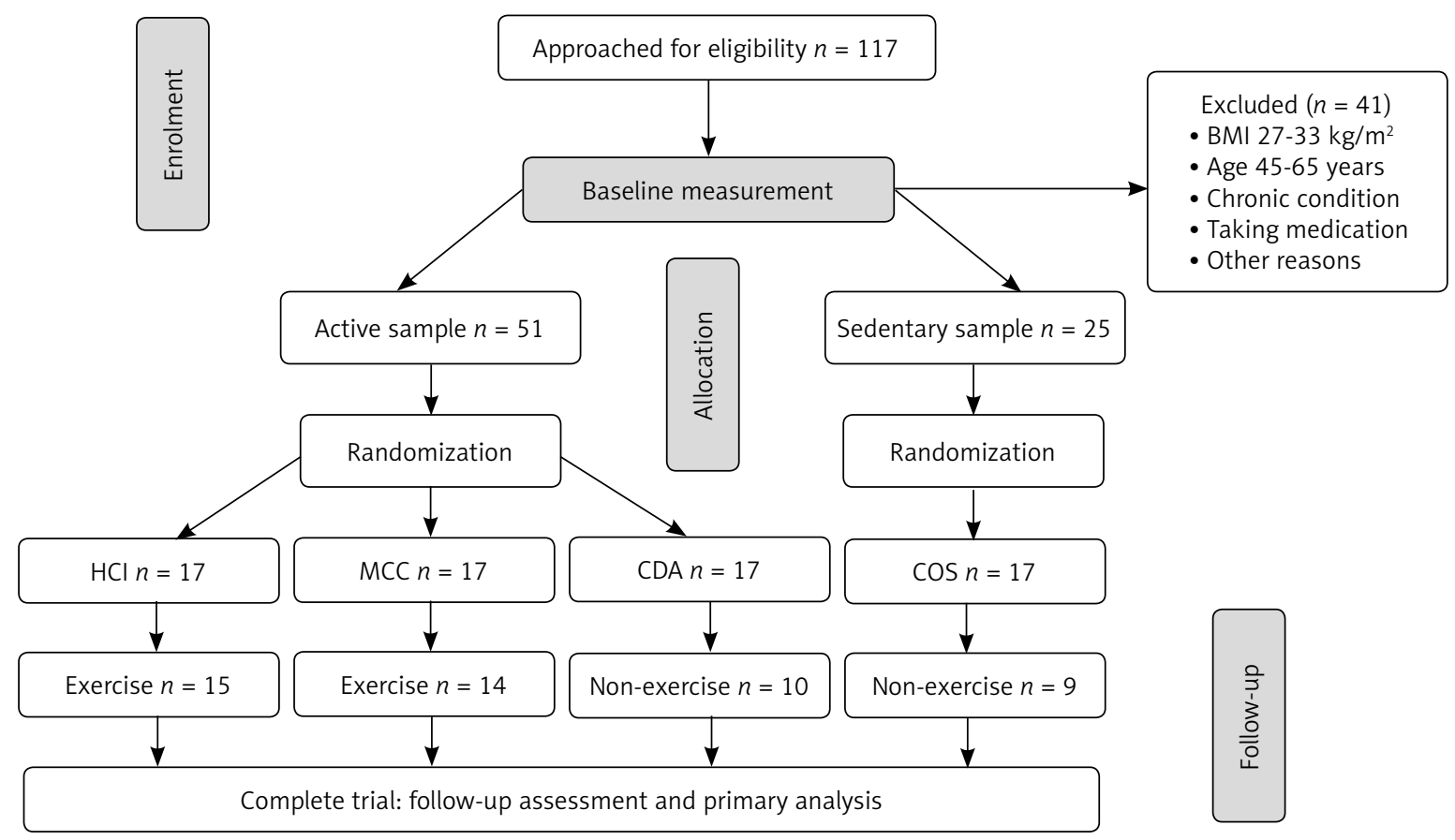

Fig. 1. Inclusion process for analysis; high-intensity concurrent interval exercise $(\mathrm{HCl})$, moderate-intensity continuous concurrent exercise (MCC), control daily active (CDA), and control sedentary (COS) 
Individual levels of physical activity were evaluated via the International Physical Activity Questionnaire (IPAQ). A detailed questionnaire was used to assess physical activity on which participants answered on a scale of 0 (none) to 10 (exercise > 1 hour four times/ week). A random selection of samples was made according to the results and they were divided into four groups: a control sedentary group ( $<5$ on the scale) and three active groups ( $\geq 5$ on the scale) [19]; high-intensity concurrent interval exercise $(\mathrm{HCl} ; n=15)$, moderate-intensity continuous concurrent exercise (MCC; $n=14)$, control daily active (CDA; $n=10)$, and control sedentary (COS; $n=9$ ) were enlisted. Participants of the control sedentary group, although physically active, did not perform any resistance training or any other type of regular exercise for 6 months before the start of the study (Fig. 1). The study followed the Declaration of Helsinki, which was approved by the research committees of the Universities of Guilan, Rasht, Iran.

\section{Research consideration}

Participants' physical activity and demographic backgrounds were determined through a series of interviews. Subsequent to gaining consent, four groups visited the clinical research center twice: at baseline, and post-test after 10 weeks. Participants' physical activity and demographic backgrounds were determined through a series of interviews. As the next step, height $(\mathrm{cm})$ and body weight $(\mathrm{kg})$ measurements were carried out by calibrated devices in a standardized manner. Next, BMI was calculated using the equation body mass/height squared. Additionally, a fasting blood sample was collected, and participants performed a 24-hour food recall. Finally, HR max measurements were carried out by a telemetric heart rate monitor (Polar Electro Oy, Kempele, Finland) and the Brzycki equation was used for the estimation of the one repetition maximum (1RM) [20]. At baseline and endpoint meas- urements were performed by physicians and exercise physiologists at the same time of day.

\section{Concurrent exercise training protocol}

The training interventions lasted for 10 weeks and the participants exercised three times per week for 50-65 min/session with a minimal interval of 48 hours between them. During the 2 weeks, both groups $(\mathrm{HCl}$ and $\mathrm{MCC}$ ) performed three times resistance and endurance concurrent training (CT) sessions. Endurance and resistance training (RT) were not done on the same days and in the same session. The $\mathrm{HCl}(\mathrm{RT}+\mathrm{HIIT})$ and MCC $(R T+C T)$ groups received either the HIIT or $\mathrm{CT}$ and RT (moderate or high training) protocols as described above; all participants expended energy up to the 500-kcal goal (Table 1).

\section{Resistance training program}

The RT protocol was used to complete a resistance circuit containing specific exercises including (upper and lower muscle groups; nine exercises) leg press, chest press, lat pull-down, leg extension, leg curl, cable crossover, biceps curl, triceps extension, and abdominal crunches. The RT program was based on the recommendations of the American College of Sports Medicine (ACSM) for RT in healthy adults including 3-4 sets, 8-15 repetitions, 30-60 second rest interval between 2 sets, and 2-3 minute rest between exercises [21]. Total training time ranged from 35 to 45 minutes, based on the number of exercises assigned (Table 1). The study group's warm-up and cool-down were performed prior to and after each session (both of 5-10 minutes duration, and consisting of walking and light, static stretching [avoiding muscle pain] in most muscle groups). In order to involve the major muscle groups, the RT procedure was followed, using a full range of motion that is considered an accurate method for the studies $[21,22]$. To avoid potential risks, as

Table 1. Resistance training program

\begin{tabular}{lcccc}
\hline Program variable & Training intensity (1RM) & Training volume & Rest time (min) & Energy expenditure (kcal) \\
\hline Weeks & \multicolumn{2}{c}{ High training } \\
\hline $1-2$ & $60-70 \%$ & $2 \times 8-10$ & 1 & 300 \\
\hline $2-4$ & $70 \%$ & $3 \times 8-10$ & 30 & 400 \\
\hline $5-10$ & $70-85 \%$ & $4 \times 8-10$ & 1 & 500 \\
\hline \multicolumn{5}{c}{ Moderate training } \\
\hline $1-2$ & $40-60 \%$ & $3 \times 12-15$ & 1 & 300 \\
\hline 2-4 & $60 \%$ & $3 \times 12-15$ & 30 & 400 \\
\hline $5-10$ & $60-70 \%$ & $4 \times 12-15$ & 1 & 500 \\
\hline RM - one repetition maximum, volume $=$ sets $\times$ repetitions
\end{tabular}




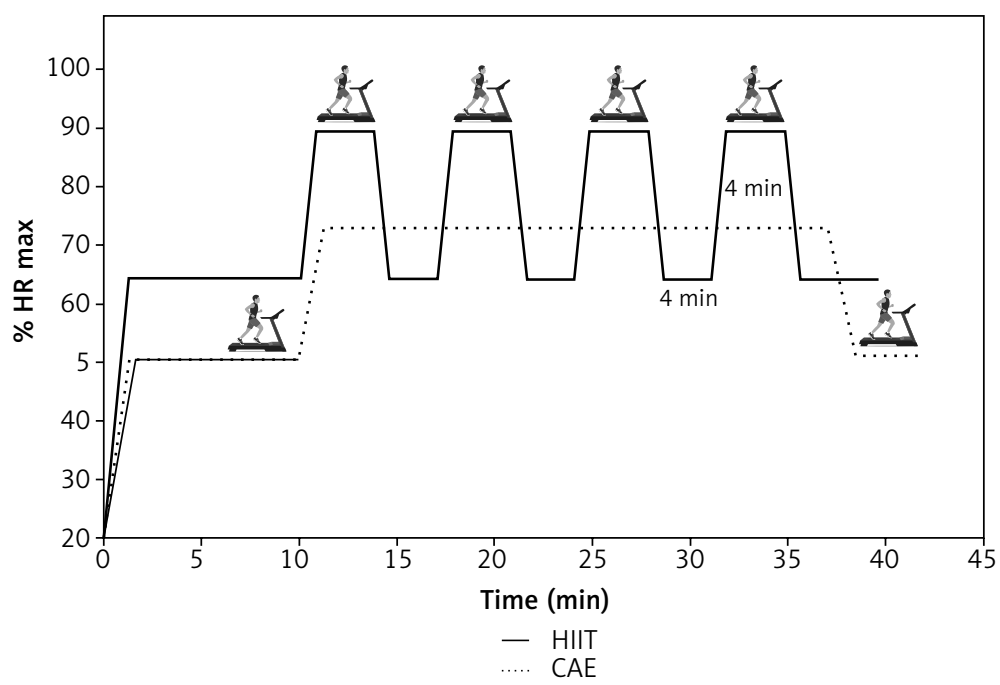

Fig. 1. Inclusion process for analysis; high-intensity concurrent interval exercise ( $\mathrm{HCl})$, moderate-intensity continuous concurrent exercise (MCC), control daily active (CDA), and control sedentary (COS)

a rule, the following activities were avoided: 1) events associated with Valsalva's mechanism, 2) ballistic and plyometric movements, and 3) positions of extreme muscular tension.

\section{Continuous aerobic training program}

The continuous aerobic training (CAT) session in cluded walking/running on a treadmill, a warm-up period of 5-10 minutes at 50\% of HR max, followed by 45 50 minutes of walking/running at $60-75 \%$ of $\mathrm{HR}$ max. Participants then completed the workout with a cooling-off period of $5-10$ minutes at $50 \%$ of $\mathrm{HR}$ max for a total duration of 45-55 minutes (Fig. 2).

\section{High-intensity interval training}

HIIT sessions began with 5-10 minutes of warm-up. Subsequently, participants were engaged in training sessions of $4 \times 4$ minutes intervals at $85-95 \% \mathrm{HR}$ max (with the target zone maintained for at least 2 minutes) interspersed with a 4-minute recovery period at $65 \%$ HR max, concluding with a cool-down (5 minutes), with an overall training time ranging between 40 and 50 minutes [22, 23] (Fig. 2).

Study subjects assigned to the control groups avoided any participation in circuit training sessions. They were requested to keep up with their routine exercises and diet throughout the study. Each training session was performed under the supervision of a researcher, to guide, aid, and to ensure the proper performance rate and load. All these sessions were carried out at Guilan Talent Discovery and Profession Athletic Center, Rasht, Iran.

\section{Magnetic resonance imaging}

Visceral adipose tissue (VAT), subcutaneous adipose tissue (SAT), and total abdominal fat volumes were measured by magnetic resonance imaging (MRI; premenopausal images: 1.5-T whole-body scanner, General Electric, Waukesha, WI; postmenopausal images: 1.5-T wholebody scanner, Philips Intera, Andover, MA). Slice thickness was $1 \mathrm{~cm}$, with approximately 12-24 consecutive slices per scan. An automatic fat separation program was used for image analysis (Slice-O-Matic, Tomovision Inc., Montreal, Canada) [24]. A thorough description and model of the methods used for various tissues segmentation are shown in a different location [28]. Every image was analyzed twice with the subject's name concealed from the operator to avoid any potential errors. A single researcher was held responsible for analyzing both pre- and post-test images. The range from the superior part of the head of the femur up to the inferior most part of the kidneys was set as the abdominal fat calculation area. The sum of overall fat contents of all slices was used to evaluate total belly fat. In order to calculate subcutaneous fat, the visceral fat value in each slice was subtracted from total fat.

\section{Blood sampling}

Blood samples ( $5 \mathrm{ml}$ at each time) were retrieved from the left antecubital vein in both pre-training, following a 12-hour overnight fast, and post-training, following a 12-hour overnight fast and a 24-hour period of no exercise. For both groups, this procedure was performed at 7:30 A.M. by the laboratory practitioner with the subject in a sitting position.

Thereafter the samples remained at room temperature for clotting purposes for about half an hour. Next, 
the samples were centrifuged at $3000 \mathrm{rpm}$ (1500 g) for 15 minutes, and plasma was removed and frozen at $-80^{\circ} \mathrm{C}$ plasma levels of the irisin levels.

Serum irisin levels were measured by enzyme immunoassay (BioVendor, USA) following the user's manual. Assay range: $0.001 \mu \mathrm{g} / \mathrm{ml}, 5 \mu \mathrm{g} / \mathrm{ml}$. Irisin levels range in human plasma and serum from $0.2 \mu \mathrm{g} / \mathrm{ml}$ to $>2 \mu \mathrm{g} / \mathrm{ml}$. Special kits, utilizing enzymatic colorimetric techniques, were used for glucose level measurements. The insulin serum level measurement was carried out via an enzyme-linked immunosorbent assay (ELISA) kit. The quantitative insulin sensitivity check index (QUICKI) was determined using the formula described by Katz et al. [25]:

QUICK $=1 /(\log ($ fasting insulin $\mu \mathrm{U} / \mathrm{ml})+\log$

(fasting glucose $\mathrm{mg} / \mathrm{dl}$ ))

\section{Statistical analyses}

The Shapiro-Wilk test was applied to test the data normality, and homogeneity of variance was examined using Levine's test. ANOVA measures were used to compare data of the four groups, using the Bonferroni method to locate means that were significantly different. Additionally, comparisons were made of variances among four groups using the independent $t$-test at a different time of measurements. Spearman's rank correlation test was used to test the relationship between insulin sensitivity, irisin level, weight loss and abdominal fat distribution. Data are expressed as mean $\pm \mathrm{SD}$; $p$-values below 0.05 were considered significant. Statistical analyses were performed using the software SPSS version 20 .

\section{Results}

\section{Pre-intervention subjects' characteristics and changes over the intervention period}

Forty-eight participants (age; $54.8 \pm 4.17$ years, weight; $72.62 \pm 8.45 \mathrm{~kg}, \mathrm{BMl} ; 29.73 \pm 3.59 \mathrm{~kg} / \mathrm{m}^{2}$ mean $\pm \mathrm{SD}$ ) completed the 10 -week program without any problems. The subjects showed tolerance to both $\mathrm{HCl}$ and MCC, and all the exercise sessions ran smoothly without any interruptions due to health issues. Baseline and post-test characteristics of the study group are summarized in Table 2. No significant differences were noted in any demographic parameter between the four groups at pre-test.

\section{Baseline changes in circulating irisin over the intervention period}

Mean baseline irisin concentration was 1.33 $\pm 0.36 \mu \mathrm{g} / \mathrm{ml}$ for $\mathrm{HCl}, 1.28 \pm 0.29 \mu \mathrm{g} / \mathrm{ml}$ for MCC, 1.35 $\pm 0.42 \mu \mathrm{g} / \mathrm{ml}$ for COD, and $1.38 \pm 0.45 \mu \mathrm{g} / \mathrm{ml}$ for COS, with no differences between groups $(p=0.61)$, as shown in Table 2. Individual changes in plasma irisin concentration illustrate the analysis of changes in parameters for the $\mathrm{HCl}$ or $\mathrm{MCC}$ cohorts [2]. Plasma irisin significantly increases by $2.20 \pm 0.42 \mu \mathrm{g} / \mathrm{ml}(p<0.001)$

Table 2. Baseline and post-training anthropometric factors, clinical characteristics and abdominal fat of subjects who underwent study

\begin{tabular}{|c|c|c|c|c|c|c|c|c|}
\hline \multirow{2}{*}{ Variable Group } & \multicolumn{2}{|c|}{$\mathrm{HCl}(n=15)$} & \multicolumn{2}{|c|}{$\operatorname{MCC}(n=14)$} & \multicolumn{2}{|c|}{$\mathrm{CDA}(n=10)$} & \multicolumn{2}{|c|}{$\cos (n=9)$} \\
\hline & Pre & Post & Pre & Post & Pre & Post & Pre & Post \\
\hline Age, years & $4.13 \pm 57.11$ & - & $54.10 \pm 5.08$ & - & $54.10 \pm 5.08$ & - & $56.66 \pm 3.92$ & - \\
\hline Weight, kg & $70.32 \pm 6.49$ & $67.38 \pm 5.87^{* *}$ & $74.45 \pm 6.17$ & $72.65 \pm 6.67^{* *}$ & $72.45 \pm 12.36$ & $74.39 \pm 11.7$ & $73.34 \pm 9.66$ & $72.88 \pm 10.88$ \\
\hline $\mathrm{BMI}, \mathrm{kg} / \mathrm{m}^{2}$ & $28.31 \pm 2.20$ & $27.00 \pm 1.80^{* *}$ & $30.62 \pm 3.08$ & $30.02 \pm 3.83^{*}$ & $29.92 \pm 4.15$ & $29.77 \pm 3.83$ & $30.48 \pm 5.15$ & $30.36 \pm 5.27$ \\
\hline WHR & $0.91 \pm 0.04$ & $0.89 \pm 0.02^{* *}$ & $0.03 \pm 0.92$ & $0.91 \pm 0.03^{*}$ & $0.91 \pm 0.02$ & $0.91 \pm 0.03$ & $0.93 \pm 0.02$ & $0.93 \pm 0.02^{a}$ \\
\hline Glucose, mg/dl & $97.00 \pm 7.24$ & $92.73 \pm 5.93$ & $94.14 \pm 7.89$ & $93.21 \pm 8.18$ & $93.60 \pm 9.89$ & $93.00 \pm 7.55$ & $99.66 \pm 10.12$ & $102.33 \pm 10.28^{a}$ \\
\hline QUICKI & $0.35 \pm 0.02$ & $0.37 \pm 0.02^{*}$ & $0.35 \pm 0.02^{*}$ & $0.36 \pm 0.02$ & $0.36 \pm 0.02$ & $0.35 \pm 0.02$ & $0.34 \pm 0.02$ & $0.34 \pm 0.02$ \\
\hline Irisin, $\mu \mathrm{g} / \mathrm{ml}$ & $1.33 \pm 0.36$ & $2.20 \pm 0.42^{\star *}$ & $1.30 \pm 0.28$ & $1.56 \pm 0.51^{a}$ & $1.35 \pm 0.42$ & $1.34 \pm 0.40^{a}$ & $1.38 \pm 0.45$ & $1.26 \pm 0.41^{\mathrm{a}}$ \\
\hline $\begin{array}{l}\text { Subcutaneous } \\
\text { abdominal fat, } \\
\mathrm{cm}^{2}\end{array}$ & $97.13 \pm 21.75$ & $93.25 \pm 20.87^{* *}$ & $102.43 \pm 21.75$ & $101.22 \pm 21.62^{*}$ & $99.44 \pm 16.33$ & $99.37 \pm 16.46$ & $108.6 \pm 31.58$ & $108.68 \pm 32.43$ \\
\hline $\begin{array}{l}\text { Visceral } \\
\text { abdominal fat, } \\
\mathrm{cm}^{2}\end{array}$ & $11.05 \pm 2.74$ & $10.14 \pm 2.62^{* *}$ & $11.90 \pm 3.05$ & $11.68 \pm 2.88$ & $11.75 \pm 1.89$ & $11.93 \pm 1.84$ & $12.31 \pm 4.02$ & $12.62 \pm 4.10$ \\
\hline
\end{tabular}

Total abdominal $105.51 \pm 24.32 \quad 94.66 \pm 19.59^{* *} 115.62 \pm 24.78 \quad 110.89 \pm 20.95 \quad 111.20 \pm 17.98 \quad 111.30 \pm 18.03 \quad 120.20 \pm 35.59 \quad 121.30 \pm 36.34$ fat, $\mathrm{cm}^{2}$

All data are presented as means \pm standard deviations; $\mathrm{HCl}$ - high-intensity concurrent interval exercise, $\mathrm{MCC}$ - moderate-intensity continuous concurrent exercise, CDA - control daily active, COD - control sedentary, BMI - body mass index, WHR - waist-to-hip ratio, QUICKI - quantitative insulin sensitivity check index, ${ }^{*}$ significant difference from pre-test to post-exercise $(p<0.05),{ }^{*}$ significant difference from pre-test to post-exercise $(p<0.001),{ }^{\text {a }}$ significant difference in COS or CDA or MCC compared to $\mathrm{HCl}$ at the same time $(p<0.05)$ 
following $\mathrm{HCl}$ training but remains unchanged after MCC training.

\section{Quantitative insulin-sensitivity check index}

The mean baseline insulin sensitivity QUICKI concentration did not differ significantly between different groups $(p=0.80)$. Two-way mixed ANOVA and Bonferroni followup test demonstrated a significant increase from pre- to post-exercise for $\mathrm{HCl}(4.71 \%, p<0.05)$ and MCC $(3.83 \%$, $p<0.05)$, as shown in Table 2 . There was no significant difference in the control groups' insulin sensitivity level $(p>0.05)$.

\section{Abdominal fat distribution (visceral vs. subcutaneous) and total abdominal fot}

Inter-group changes indicated that subcutaneous abdominal fat was significantly decreased in $\mathrm{HCl}$ and MCC groups (3.99\%; $p<0.001$ and $1.18 \% ; p=0.011$, respectively). There was no significant difference in the control groups' subcutaneous abdominal fat $(p>0.05)$. The inter-group evaluation showed a significant reduction for visceral and total abdominal fat in the $\mathrm{HCl}$ group $(8.27 \%, 10.27 \% ; p<0.001$, respectively), as shown in Table 2. It should also be noted that there was no significant difference between the MCC, COS and CDA groups in visceral and total abdominal fat.

\section{Discussion}

The results of the present study suggest that postmenopausal active women can improve most of the metabolic health-related parameters by concurrently undergoing HIIT and RT. Moreover, the extent of the improvements achieved seems to be affected by volume and/or intensity of the endurance training because different gains were observed for the RT + CT (MCC) and RT + HIIT ( $\mathrm{HCl}$ ) groups.

To our knowledge, this study is the first of its kind to make a comparison between the influences of $\mathrm{HCl}$, MCC, along with two control groups in a 10-week training period, on serum irisin level, insulin sensitivity status and abdominal fat in postmenopausal women.

The new findings of the current study include promising effects that $\mathrm{HCl}$ promoted increased plasma levels of irisin, and decreased visceral, subcutaneous and total abdominal fat. Additionally, the increased QUICKI decreased with a positive change in body fat, visceral abdominal fat compared to the MCC group.

One must acknowledge that various concurrent training protocols have been examined in this study. Endurance and RT do not have to be executed on the same day and in the same session. The relative improvement in blood irisin observed in the $\mathrm{HCL}$ group could be partially due to the training sequence. However, not all studies report considerable changes [12, $26,27]$ or decline $[28,29]$ in baseline irisin concentrations, whereas studies on older subjects presented mixed results [16, 28]. Some studies suggest that the release of irisin as a myokine into the circulation during training may be an endocrine function of the body to control metabolism. Numerous studies have demonstrated that exercise drives irisin secretion in the skeletal muscle that leads to the expression of FNDC5 protein, thus elevating the irisin formation levels [30]. Once in circulation, this myokine transforms white adipose tissue (WAT) into brite (brown in white)/beige fat cells by upregulating the genes responsible for changing with browning (such as uncoupling protein UCP-1, peroxisome proliferator-activated receptor $\gamma-$ PPAR $\gamma$, and PR domain containing 16 - PRDM16) [31]. Once converted from white to brown, adipose tissue takes on the function of dispersing heat energy, while white adipose tissue is responsible for storing energy. This event, in turn, causes an increase in total body energy expenditure [32].

Therefore, a rise in irisin and FNDC5 levels in response to $\mathrm{HCL}$ would be rational as it is largely characterized by heightened oxidative capacity and mitochondrial functions. Such influences of irisin are probably facilitated by pathways associated with phosphorylation of p38 mitogen-activated protein kinase (p38 MAPK) and extracellular signal-regulated kinase (ERK) [33]. An increase in energy expenditure and glucose homeostasis is a good reflection of irisin's role in metabolism [6].

The transformed beige adipocytes are specific for heat generation and energy expenditure due to their rich mitochondrial UCP-1 content [34]. Irisin upregulates lipolysis related genes, such as hormone-sensitive lipase (HSL), adipose triglyceride lipase (ATGL), and fatty acid binding protein 4 (FABP4), ultimately increasing glycerol release and reducing lipid accumulation in adipocytes. Furthermore, the glucose uptake capacity of beige fat cells is also boosted as their glucose transporter type 4 (GLUT4) expression increases.

Taken together, our findings support the hypothesis that insulin sensitivity of peripheral tissues could be more closely linked to irisin values, specifically in striated muscles. This is a difference that can be detected even in basal conditions, independently of physical activity. Irisin, as a myokine secreted by striated muscles, has been known to shift glucose for energy expenditure in muscles to maintain glucose homeostasis. Moreover, insulin resistance associated with aging has also been linked to abdominal obesity [35] and Rice et al. [36] suggested that improved insulin sensitivity observed after resistance exercises may decrease visceral and abdominal subcutaneous adipose tissue or abdominal obesity. In the present study, $\mathrm{HCl}$ led to reductions in both abdominal fat distribution (visceral vs. subcutaneous) and total 
abdominal adipose tissue (Table 2). Thus, it can be said that equipped with such extraordinary properties, irisin perhaps is a strong likely candidate to be used against obesity and likewise other metabolic conditions.

A limitation of the present study which should be noted is the small sample size in control and experimental groups at the end of the experiment. Therefore, further studies with a large sample size are needed to clarify the relationship between irisin and insulin resistance in postmenopausal women.

One of the most significant steps in any future investigation is to determine whether irisin has a receptor for characterizing its mode of action. This would aid us in our understanding of irisin's functions as well as the cascading signals involved in its effect. Likewise, it is important to provide a clear explanation of the mechanism of how muscle tissue secretes irisin during exercise. Such information will be crucial in explaining what distinguishes the effects of acute and chronic training on irisin blood levels, and to discover whether there are other stimulants of irisin release.

\section{Conclusions}

According to the results, the $\mathrm{HCl}$ results in an increased irisin level and decreased visceral abdominal fat. Also, the insulin sensitivity status was significantly increased, and subcutaneous abdominal fat significantly decreased in the MCC group. In contrast, the MCC did not produce a significant effect on irisin level, and decreased visceral and total abdominal fat.

Finally, we hypothesized that active participants in the HIC group would experience a greater response to irisin circulation, in addition to visceral, subcutaneous, and total abdominal fat and QUICKI after 10 weeks, compared to the MCC group. This may be of great significance when it comes to designing an optimal training program for women experiencing menopause.

\section{Acknowledgments}

This article has been taken from the first researcher's Ph.D. dissertation at the University of Guilan. We are grateful to the faculties of physical education and sport science as well as the participants in this research.

\section{Disclosure}

The authors report no conflicts of interest.

\section{References}

1.Kozakowski J, Gietka CM, Leszczyńska D, Majos A. Obesity in menopause our negligence or an unfortunate inevitability? Prz Menopauzalny 2017; 16: 61-65.

2.Lee IM, Shiroma EJ, Lobelo F, et al. Effect of physical inactivity on major non-communicable diseases worldwide: an analysis of burden of disease and life expectancy. Lancet 2012; 380: 219-229.

3.Velders M, Diel P. How sex hormones promote skeletal muscle regeneration. Sports Med 2013; 43: 1089-1100.

4.Mourier A, Gautier JF, De Kerviler E, et al. Mobilization of visceral adipose tissue related to the improvement in insulin sensitivity in response to physical training in NIDDM: effects of branched-chain amino acid supplements. Diabetes Care 1997; 20: 385-391.

5.Pekkala S, Wiklund PK, Hulmi JJ, et al. Are skeletal muscle FNDC5 gene expression and irisin release regulated by exercise and related to health? J Physiol 2013; 591: 5393-5400.

6.Boström P, Wu J, Jedrychowski M, et al. A PGC1 dependent myokine that drives brown-fat-like development of white fat and thermogenesis. Nature 2012; 7382: 463-468.

7.Szumilewicz A, Worska A, Piernicka M, et al. Acute post exercise change in circulating irisin is related to more favorable lipid profile in pregnant women attending a structured exercise program and to less favorable lipid profile in controls: an experimental study with two groups. Int J Endocrinol 2019; 2019: 1932503.

8.Tsuchiya Y, Mizuno S, Morii I, Goto K. Irisin response to down-hill running exercise in humans. J Exerc Nutrition Biochem 2018; 22: 12-17.

9.Qiu S, Bosnyák E, Treff G, et al. Acute exercise-induced irisin release in healthy adults: Associations with training status and exercise mode. Eur J Sport Sci 2018; 18: 1226-1233.

10. Archundia HC, Macias CM, Ruiz M et al. Muscle irisin response to aerobic vs HIIT in overweight female adolescents. Diabetol Metab Syndr 2017; 9: 101.

11. Gamas L, Matafome P, Seiça R. Irisin and myonectin regulation in the insulin-resistant muscle: implications to adipose tissue: muscle crosstalk. J Diabetes Res 2015; 2015: 359159.

12. Angadi SS, Mookadam F, Lee CD, et al. High-intensity interval training vs. moderate-intensity continuous exercise training in heart failure with preserved ejection fraction: a pilot study. J Appl Physiol 2014; 119: 753 758.

13. Huh JY, Mougios V, Skraparlis A, et al. Irisin in response to acute and chronic whole body vibration exercise in humans. Metabolism 2014; 63: 918-921.

14. Daskalopoulou SS, Cooke AB, Gomez YH, et al. Plasma irisin levels progressively increase in response to increasing exercise workloads in young, healthy, active subjects. Eur J Endocrinol 2014; 171: 343-352.

15. Erickson HP. Irisin and FNDC5 in retrospect: an exercise hormone or a transmembrane receptor? Adipocyte 2013; 2: 289-293.

16. Scharhag RF, Meyer T, Wegmann M, et al. Irisin does not mediate resistance training- induced alterations in resting metabolic rate. Med Sci Sports Exerc 2014; 46: 1736-1743.

17. Timmons JA, Baar K, Davidsen PK, Atherton PJ. Is irisin a human exercise gene? Nature 2012; 7413: 463-468.

18. Khodadadi H, Rajabi H, Attarzadeh SR, et al. The effect of high-intensity interval training (HIIT) and pilates on levels of irisin and insulin resistance in overweight women. Iran J Endocrinol Metab 2014; 16: 190-196.

19. García FB, Reyes GR, Morales SS, et al. Relationship between myostatin and irisin in type 2 diabetes mellitus: a compensatory mechanism to an unfavorable metabolic state? Endocrine 2016; 52: 54-62.

20. Brzycki M. Strength testing predicting a one-rep max from reps to fatigue. J Phys Educ Recreat Dance 1993; 64: 88-90.

21. Medicine ACoS. American College of Sports Medicine position stand. Progression models in resistance training for healthy adults. Med Sci Sports Exerc 2009; 41: 687-708. 
22. Francois ME, Durrer C, Pistawka KJ, et al. Combined interval training and post- exercise nutrition in type 2 diabetes: a randomized control trial. Front Physiol 2017; 8: 52.

23. Bond B, Cockcroft EJ, Williams CA, et al. Two weeks of high-intensity interval training improves novel but not traditional cardiovascular disease risk factors in adolescents. Am J Physiol Heart Circ Physiol 2015; 309: $\mathrm{H} 1039-\mathrm{H} 47$.

24. Shen W, Chen J. Application of imaging and other noninvasive techniques in determining adipose tissue mass. Methods Mol Biol 2008; 456: 39-54.

25. Katz A, Nambi SS, Mather K, Baron AD, et al. Quantitative insulin sensitivity check index: a simple, accurate method for assessing insulin sensitivity in humans. J Clin Endocrinol Metab 2000; 85: 2402-2410.

26. Askari H, Rajani SF, Poorebrahim M, et al. A glance at the therapeutic potential of irisin against diseases involving inflammation, oxidative stress, and apoptosis: an introductory review. Pharmacol Res 2018; 129: 44-55.

27. Gamas L, Paulo M, Seiça R. Irisin and myonectin regulation in the insulin-resistant muscle: implications to adipose tissue: muscle crosstalk. J Diabetes Res 2015; 2015: 359159.

28. Tibana R, da Cunh ND, De Souza NF, et al. Irisin levels are not associated to resistance training-induced alterations in body mass composition in older untrained women with and without obesity. J Nutr Health Aging 2017; 21: 241-246

29. Moienneia N, Hosseini SRA. Acute and chronic responses of metabolic myokine to different intensities of exercise in sedentary young women. Obes Med 2016; 1: 15-20.

30. Panati K, Suneetha Y, Narala V. Irisin/FNDC5 an updated review. Eur Rev Med Pharmacol Sci 2016; 20: 689-697.

31. Zhang $\mathrm{Y}$, Xie C, Wang H, et al. Irisin exerts dual effects on browning and adipogenesis of human white adipocytes. Am J Physiol Endocrinol Metab 2016; 311: 530-541.

32. Shirvani H, Rahmati-Ahmadabad S. Irisin interaction with adipose tissue secretions by exercise training and flaxseed oil supplement. Lipids Health Dis 2019; 18: 15-24.

33. Zhang Y, Li R, Meng Y, et al. Irisin stimulates browning of white adipocytes through mitogen-activated protein kinase p38 MAP kinase and ERK MAP kinase signaling. Diabetes 2014; 63: 514-525.

34. Kong X, Zhou P, Yao T, Kazak L, et al. Thermoneutrality induces skeletal muscle myopathy via brown adipose tissue in an IRF4, and myostatin dependent manner. bioRxiv 274365; doi: https://doi.org/10.1101/274365

35. Kohrt WM, Kirwan JP, Staten MA, et al. Insulin resistance in aging is related to abdominal obesity. Diabetes 1993; 42: 273-281.

36. Rice B, Janssen I, Hudson R, Ross R. Effects of aerobic or resistance exercise and/or diet on glucose tolerance and plasma insulin levels in obese men. Diabetes Care 1999; 22: 684-691. 